\title{
Object Oriented Implementation of Fuzzy Logic Systems
}

\author{
Radim Belohlavek, Antonin Dvorak, David Jedelsk)J and Vilem \\ Novak \\ University O/ Ostrava, Institute tor Research and Applications O/ \\ Fuzzy Modeling \\ Bralova 7, 70103 Ostrava 1, Czech Republic \\ e-mail: \{belohlav.dvoraka.jedelsky.novakv\}@osu.cz
}

\begin{abstract}
Various aspects of implementation of fuzzy software systems are discussed. Some theoretical considerations on basic notions are introduced and also ouf approach to implemetation is presented.
\end{abstract}

Keywords:

Fuzzy control, object-oriented programming.

\section{INTRODUCTION}

This contribution has two main aims: to present main concepts which lead us to the creation of the software system LFLC (Linguistic Fuzzy Logic Controller) for fuzzy control, decision making and modeling, and to discuss object-oriented implementation ofthese concepts. We believe that ouf experience can be useful for those who work in the field of the soft computing software and, also to advanced users who want to understand better the theoretical background ofthe fuzzy control and modeling. We use $\mathrm{C}++$ for programming and will use the common terminology used by the $\mathrm{C}++$ programmers. The following discussion will concern only implementation of the kernel of fuzzy software system, i.e. basic classes and their data members and methods. We will not discuss the design ofuser interface.

\section{LINGUISTICALLY ORIENTED FUZZY CONTROL AND MODELING}

Fuzzy sets are intended for modeling of situations where the phenomenon of vagueness is present. A typical case is modeling of the semantics of natural language descriptions (e.g. descriptions of complex systems). Recall that a fuzzy set in the universe set $U$ is a mapping $A: U->[0,1]$. For XE $U$, the value $A(x)$ is interpreted as the truth degree of " $\mathrm{x}$ is a member of $A$ ".

\footnotetext{
The original version of this chapter was revised: The copyright line was incorrect. This has been corrected. The Erratum to this chapter is available at DOI: 10.1007/978-0-387-35390-6_58 
There are two basic kinds of representation of a fuzzy set-by the membership function or by the family of its $\alpha$-cuts (Klir et al. 1995). Similarly as in the classical case, various operations may be performed with fuzzy sets (Klir et al. 1995), based usually on the operations on the set of truth values (t-norms, t-conorms and derived operations). The most successful applications of fuzzy sets are in fuzzy control.

The role of fuzzy controllers is nowadays well acknowledged. Their idea has been proposed by L. A. Zadeh (Zadeh 1975) and it has been applied to control by E. H. Mamdani. Recall basic facts: Even in situations where no exact mathematical description of the controlled process exists, experienced operators are still able to perform successfully the control. The operators are able to express the control strategy in the form of a linguistic description. Having a suitable formal apparatus for modeling of this linguistic description at disposal we are able to mimic the decision processes made by humans. The apparatus is provided by fuzzy set theory and theory of approximate reasoning. However, the linguistic point of view gets often lost. As a result, the declared transparency of the model decreases in such case. Especially nontransparent are techniques of adaptation of the controller where the fuzzy sets representing the linguistic expressions are arbitrarily modified. This is useful from the control-engineering point of view, however, the resulting model can be hardly interpreted on the level of linguistic descriptions.

The linguistic point of view is consistently pursued in the so called linguistically oriented fuzzy control (LOFLC) (Novák 1995). The main idea of LOFLC is that of keeping in mind two levels - the level of linguistic descriptions (syn$\operatorname{tax}$ ) and the level of the meaning of the descriptions (semantics). These two levels are connected as described below. However, we insist on the fact that at every time moment, the level of syntax should have the corresponding reflection in the level of semantics and, conversely (which is crucial), the level of semantics should have the corresponding reflection in the level of syntax. The formal agenda goes as follows. We suppose that the expert knowledge of the control is expressed by the set

$\mathcal{R}=\left\{\mathcal{R}_{1}, \ldots, \mathcal{R}_{n}\right\}$

of linguistic control rules $\mathcal{R}_{i}$. The set of rules is called the linguistic description of the control. According to the information involved and the kind of the control action, $P, P I, P D$ and $P I D$ control is usually distinguished. For example, in the case of $P I$ control each $\mathcal{R}_{i}$ has the form*

$\mathcal{R}_{i}:=$ IF $e$ is $\mathcal{A}_{i}^{e}$ AND $\Delta e$ is $\mathcal{A}_{i}^{\Delta e}$ THEN $\Delta u$ is $\mathcal{B}_{i}^{\Delta u}$.

Here, $e=y-v(y$ is the process output and $v$ is the setpoint) and $\Delta e$

* Note that in the case of the so called Takagi-Sugeno models the THEN-part of the rule has the form of an arithmetic expression. 
denote the error and change of error (with respect to the previous time step), respectively, and the symbols $\mathcal{A}_{i}^{e}, \mathcal{A}_{i}^{\Delta e}, \mathcal{B}_{i}^{\Delta u}$ denote appropriate linguistic expressions. The variables $e, \Delta e$, and $y$ are the so called fuzzy variables (Zadeh 1975). The general form of the considered linguistic expressions is

$\mathcal{A}:=[\langle$ linguistic modifier $\rangle]\langle$ atomic term $\rangle$.

Here, 〈linguistic modifier) is an intensifying adverb with narrowing or extending effect (we use extremely, highly, very, rather, more or less, roughly, medium, quite roughly, very roughly, about, approximately and the negation not). The 〈atomic term〉 may be an adjective (we use smoll, medium, big), a fuzzy number or some special term (e.g. undefined). A typical example of the linguistic expression $\mathcal{A}$ is very small, roughly big etc. We described the level of linguistic descriptions. Below we describe the level of the meaning of the descriptions.

Each of the linguistic expressions is an example of the so called evaluating expressions, i.e., linguistic expressions, the meaning of which can be modelled by a fuzzy set on an ordered scale (usually on the set of real numbers). The fuzzy set representing the expression $\mathcal{A}$ is denoted by $A$. To get the meaning $A$, three facts have to be known. First, each variable involved has some context (Novák 1995). The same word may have different meanings in different contexts (consider e.g. the word small in the context of everyday temperatures and in the context of air pressures). In our conception, the context is modeled by a real interval of possible values of the particular variable. Second, there is a rule assigning to every atomic term a subset in the context of the respective variable. For instance, for the adjectives small, medium and big we use the quadratic fuzzy sets of type $S^{-}, \Pi$ and $S^{+}$, respectively. Third, the meaning of linguistic modifiers is represented by a suitable modification of the fuzzy set which represents the atomic term to which the modifier is applied.

Note that the transparent relation between the level of syntax and semantics is kept even in adaptation of the whole model due to the fact that the adaptation techniques reflect both of these levels.

To be able to derive decisions (control actions) we need a suitable inference method. Two kinds of methods are elaborated in our conception. As these methods are more or less known and are described elsewhere (Klawonn et al. 1996), (Novák 1994) we give only the final formula for the case of $P I$ control.

Given the description of actual error and its change represented by the fuzzy sets $A^{\prime e}, A^{\prime \Delta e}$, the conclusion $B^{\prime \Delta u}$ may be obtained either by the well known Max-Min (Max-t-norm) rule (Klir et al. 1995) or by the fuzzy logic inference:

$$
B^{\prime \Delta u} y=\bigvee_{x_{1}, x_{2}}\left(\left(A^{\prime e} x_{1} \wedge A^{\prime \Delta e} x_{2}\right) \otimes \bigwedge_{i=1}^{n}\left(\left(A_{i}^{e} x_{1} \wedge A_{i}^{\Delta e} x_{2}\right) \rightarrow B_{i}^{\Delta u} y\right)\right)
$$


where $\otimes$ is the operation of Eukasiewicz product and $\rightarrow$ that of the corresponding residuation.

To derive a concrete control action from the output fuzzy set, a suitable defuzzification method has to be employed (Klir et al. 1995).

\section{DESIGN OF LFLC}

In this section we present a more technical description of the basic fuzzy and linguistic concepts briefly described in previous section. The analysis of the approximate reasoning leads us to realize that there are three most important notions, namely the fuzzy set, the linguistic settings and the linguistic description. Each of these notions has a corresponding counterpart in the implementation, namely the class. These classes are not at the same level of generality. Instances of the formers are members of the latters.

\subsection{Representation of fuzzy set}

The first important class is CFuzzySet. Our intention was to make representation of fuzzy sets as general as possible. The purpose of this approach is to stress not only the properties of the fuzzy sets used in approximate reasoning. We were led to the creation of one fundamental class CFuzzySet which serves as the basis for all possible types of fuzzy sets.

All fuzzy sets have two common properties, namely the context and the membership function. CFuzzySet implements the context as a data member, being a multidimensional interval with special means for the most often used onedimensional case. The membership function is not included in CFuzzySet, but every type of fuzzy set (multidimensional, discrete, parametrically defined and fuzzy sets represented by means of $\alpha$-cuts) has implementation of its membership function in the corresponding derived classes. Types of fuzzy sets represented by classes derived from CFuzzySet are:

- Discrete fuzzy sets (class CFuzzyDiscreteSet) which are widely used in the inference routines. They are, of course, also multidimensional with onedimensional fuzzy sets as special case.

- Parametrically defined families fuzzy sets (classes CFuzzyTriangleSet, CFuzzyTrapezoidalSet and CFuzzyQudraticSet) which are most often used as meanings of atomic linguistic terms.

- Fuzzy sets represented by means of their $\alpha$-cuts (class CFuzzyAlphaCutSet).

Further, several operations on fuzzy sets are implemented - intersection, union, implication, difference etc. These operations on fuzzy sets are realized using operations on real numbers, e.g. various kinds of t-norms and t-conorms. 
Thus, for example, intersection of fuzzy sets has no preferred hidden t-norm behind, but it is possible to use minimum, product, Lukasiewicz t-norm or other t-norms as well.

\subsection{Representation of semantics of linguistic expressions}

The main idea here is to permit the same linguistic expression to have different meanings dependly on the user's choice of atomic terms and linguistic modifiers. We use the hierarchical structure of classes $C * * *$ Settings with all the necessary information for obtaining the meaning of linguistic expressions which have the form described in Section 2. Base class CSettings contains only the information common for all types of semantics, namely the linguistic context and the virtual method GetMeaning, which returns a discrete fuzzy set - meaning of an actual linguistic expression. This method has to be implemented in all the derived classes. Derived classes have the following structure:

- CBasicSettings - base class for semantics which atomic terms are parametrically defined families of fuzzy sets:

- CLinguisticSettings - atomic terms are fuzzy sets with quadratic membership functions,

- CTriangSettings - triangular membership functions,

- CTrapezoidSettings - trapezoidal membership functions.

- CLinCSettings - class representing the structure of succedent in TakagiSugeno fuzzy models.

Names and meanings of atomic terms and linguistic modifiers are placed as lists in the class CBasicSettings. There is also method GetFuzzyConst which returns the meaning of fuzzy number. The class CLinguisticSetings has special position among the family of $\mathrm{C} * * *$ Settings because atomic terms with quadratic meanings and linguistic modifiers based on horizon shifting (Novák et al. 1998) have several specific properties. List of atomic terms placed in CBasicSettings serves here as list of user defined terms.

\subsection{Representation of the linguistic descriptions}

Our idea for representation of the linguistic description is to have one class which includes not only the information concerning the meanings of all the linguistic expressions appearing in the description, but also the structure of antecedent and inference and defuzzification methods. In other words, all the data needed for the execution of inference should be here. 
The information about meanings of the linguistic expressions is stored in a matrix. The rows of the matrix correspond to the particular rules of the linguistic description, the columns correspond to individual fuzzy variables and are represented by the class CFuzzyVariable. The most important data member of this class is an instance of $\mathrm{C} * * *$ Settings. It follows that, in general, every fuzzy variable can have different interpretation of the semantics of the linguistic expressions. Furthermore CFuzzyVariable contains the name of variable and its discretization. The column itself is the list where each member contains linguistic expression and the corresponding meaning. The meaning is usually fuzzy set but for fuzzy models of Takagi-Sugeno type it is an array of real numbers - coefficients of linear combination, which are necessary for an evaluation of arithmetical expression in the succedent part of rules.

\section{ACKNOWLEDGEMENT}

This work has been supported by the grant 201/96/0985 of the GAČR.

\section{REFERENCES}

Klawonn, F. and Novák, V. (1996) The Relation between Inference and Interpolation in the Framework of Fuzzy Systems. Fuzzy Sets and Systems, 81, 331-354.

Klir, G.J. and Yuan, B. (1995) Fuzzy Sets and Fuzzy Logic. Theory and Applications. Prentice Hall, Englewood Cliffs.

Novák, V. (1992) The Alternative Mathematical Model of Linguistic Semantics and Pragmatics. Plenum, New York.

Novák, V. (1994) Fuzzy Control from The Logical Point of View. Fuzzy Sets and Systems, 66, 159-173.

Novák, V. (1995) Linguistically Oriented Fuzzy Logic Controller and Its Design. International Journal of Approximate Reasoning, 12, 263-277.

Novák, V. and Perfilieva, I. (1998) Evaluating Linguistic Expressions and Functional Fuzzy Theories in Fuzzy Logic. To appear in Computing with Words in Systems Analysis (eds. L.A. Zadeh and J. Kacpryk), Springer-Verlag, Heidelberg.

Zadeh, L.A. (1973) Outline of a New Approach to the Analysis and Synthesis of Complex Systems and Decision Processes. IEEE Transactions on Systems, Man, and Cybernetics, 1, 28-44.

Zadeh, L.A. (1975) The concept of a linguistic variable and its application to approximate reasoning I, II, III. Information Sciences, 8, 199-257, 301-357; 9, 43-80. 